\title{
Journal of Real-Time Image Processing—second issue of volume 7
}

\author{
Matthias F. Carlsohn $\cdot$ Nasser Kehtarnavaz
}

Published online: 8 May 2012

(C) Springer-Verlag 2012

JRTIP has been experiencing a huge increase in the number of manuscript submissions, which is mostly due to the increase in its recognition level. As a solution to address this issue, Springer has allowed us to increase the number of pages for the next three years starting in 2013 to 320 pages. Our current annual page count is sitting at 256 pages.

As a step to improve the review process, Springer has started to offer reviewers "incentives" for providing quality reviews. This reviewer reward program involves two incentives: (1) those reviewers who provide 5 quality reviews will be given a "certificate", and (2) a complimentary Springer book will be provided to such reviewers in acknowledgement of their fine academic service to the journal. This program has been communicated to existing reviewers stating that they need to enter 5 or more research or expertise areas into the online review system Editorial Manager.

The handling Associate Editors have been requested to enter a ranking of reviews based on the following criteria: thoroughness of comments with adequate technical details-for revisions or resubmissions providing specific and detailed comments; professional critique language free from subjective and non-technical comments; specifics on grammatical weaknesses and suggestions for improvements; adequate cross-checking with the state-of-the-art references; and alerting self-plagiarism or any evidence of

M. F. Carlsohn ( $\square)$

Computer Vision and Image Communication Engineering and Consultancy Dr. Carlsohn, Bremen, Germany

e-mail: matthias.carlsohn@t-online.de

\section{N. Kehtarnavaz}

Department of Electrical Engineering,

University of Texas at Dallas,

Dallas, USA

e-mail: kehtar@utdallas.edu plagiarism to the handling editor. Questions regarding this reward program should be directed to Hari Gopakumar (Hari.Gopakumar@springer.com).

Another recent initiative which is worth mentioning is availability of free downloads from the JRTIP website http://www.springer.com/11554 of the five most downloaded articles.

The editorial board of JRTIP met in Brussels where they were attending the 2012 SPIE Conference on Real-Time Image and Video Processing. A picture of some of the editorial board members present at the Springer-sponsored dinner meeting is shown below. In this meeting, various issues related to improving the operation of JRTIP were discussed. The editorial board will have its next meeting in San Francisco in February 2013 where the SPIE Conference on Real-Time Image and Video Processing will take place as part of the SPIE Electronic Imaging Symposium. We encourage submission of papers to this conference, which is solely dedicated to real-time aspects of image and video processing.

This regular issue includes five papers covering the fields of stereo image processing, pose estimation in 3D imaging, spectral imaging and wavelet transform applications.

As the previous issue of JRTIP was dedicated to 3D real-time image processing the first three papers continue this theme.

The first paper by Gutemberg Bezerra Guerra-Filho et al. deals with the challenging performance requirements for stereo matching in 3D reconstruction of high-resolution images examining the computational complexity supported by run-time experiments and contributing a dense stereo matching algorithm including real-time performance on real and synthetic data.

The second paper by McCullagh discusses the computational power of the Cell Broadband Engine, a new 


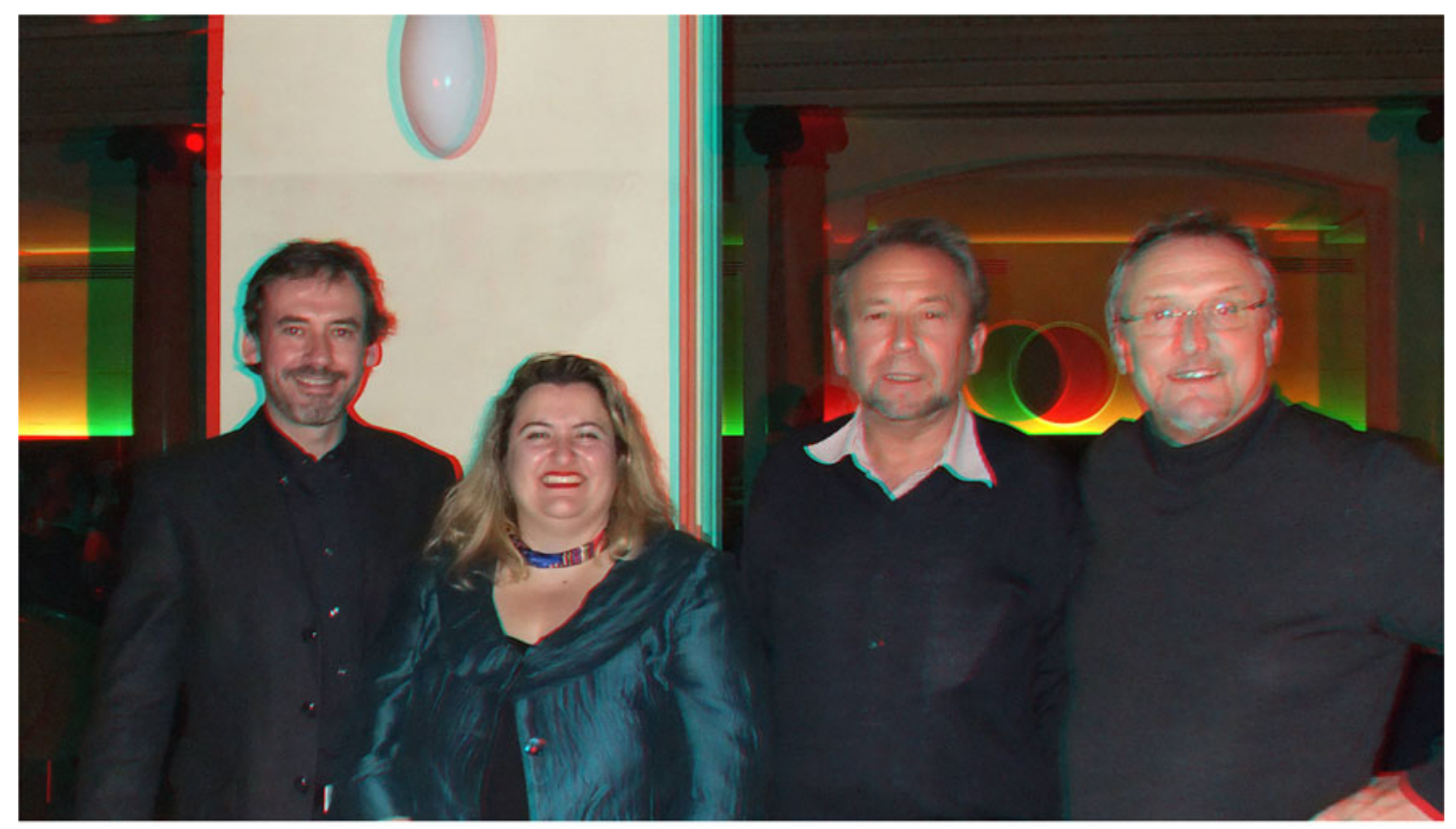

Left to right: Luis Salgado, Rube Mehrubeoglu, Volodymyr Ponomaryov, Matthias Carlsohn (as red/cyan anaglyph for 3D display)

processor released by several multimedia and computer brands implementing a disparity map computation for stereo imaging in real-time.

The next paper presented by Grest and his co-authors provides a comparison of different methods for the estimation of orientation and position of objects using OpenCV with regard to their reliability and adjustable accuracy for increased throughput in order to meet realtime deadlines.

The fourth paper deals with the multi-dimensionality in real-time image processing within the context of hyperspectral imaging, where Chen et al. present four real-time implementations of N-FINDR addressing techniques to cope with: number of endmembers, use of random initial endmembers, dimensionality reduction and finally computational costs caused by an exhaustive search for endmembers.

The final paper of Pande and Zambreno presents a lightweight multimedia encryption strategy based on a modified Discrete Wavelet Transform providing joint multimedia encryption and compression to avoid increases in computation costs leading to tradeoffs in system latency, throughput, hardware requirements and power usage of embedded real-time imaging systems. 